\title{
Misuse of the Wooden Tongue Depressor
}

Sir,

The purpose of this review is to sound a red alert over the erroneous use of disposal wooden tongue depressor (DWTD) for high risk patients. A literature search was conducted for medical and nonmedical uses of the disposable wooden tongue depressor (DWTD). Search strategy was through Medline and Google.

Besides inspecting throats, medical uses include mixing medications, splinting fractures, securing intravenous fluid lines, handling stool specimens. Nonmedical uses of the DWTD include bookmark, toy, ruler, memo and phone pad, recording housestaff evaluations, spoon for ice cream, mixing hot beverages, and a toothpick. However, use of the DWTD, is not without associated morbidity and mortality, especially in the immunocompromised host. There are scattered reports, in the literature, of nosocomial infection with Rhizoporus microsporum, the fungus responsible for mucormycosis, particularly after the use of DWTDs in patients with depressed immunity. ${ }^{1-3}$ Mucormycosis is an opportunistic fungal infection. Innocent as it may seem, inspecting throats of immunocompromised patients with a DWTD, or even using it to mix medications, is a risk factor for fungal spore transmission. ${ }^{1,2}$ In addition to their weak immune defences, premature babies are at high risk because of the use of occlusive dressings, and humidity inside the incubators. In premature infants, DWTDs serve as a vehicle for fungal spore transmission, when used as splints to secure lines for intravenous and intraarterial fluids. ${ }^{3}$ In a nosocomial mucormycosis infection in an
Intensive Care Unit in Spain, the fungus was even grown from unopened DWTD boxes. ${ }^{4}$

In conclusion, the use and abuse of the DWTD were reviewed. Associated morbidity and mortality occurs in immune deficiency states, which promote fungal proliferation. Prevention of infection includes avoidance of the DWTDs and other wooden instruments, when caring for a patient with depressed immunity. While the DWTD may be used safely in healthy children, it should be avoided in high risk patients, such as the immunocompromized and neonates.

Kamal F. Akl MD

Assistant Professor of Pediatrics - Consultant Pediatric Nephrologist-Jordan University Hospital-College of Medicine- University of Jordan. Box 831373 Amman 11183 - Jordan

E-mail:kachbl@yahoo.com

[DOI-10.1007/s12098-010-0026-0]

\section{REFERENCES}

1. Holzel H, Macqueen S, MacDonald A et al. Rhizopus microsporus in wooden tongue depressors : a major threat or minor inconvenience? J Hosp Infect 1998; 38: 113-118.

2. Simbli M, Hakim F, Koudieh M, Tleyjeh IM. Nosocomial post-traumatic cutaneous mucormycosis : a systemic review. Scan J Infect Dis 2008; 40 : 577-582.

3. Mitchel SJ, Gray J, Morgan ME, Hocking MD, Durbin GM. Nosocomial infection with Rhizopus microsporus in preterm infants : association with wooden tongue depressors. Lancet 1996; 348: 441-443.

4. Maravi-Poma E, Rodreguiz - Tudela JL, de Jalon JG et al. Outbreak of gastric mucormycosis associated with use of wooden tongue depressors in critically ill patients. Intensive Care Medicine 2004; 30: 724-728.

\section{Retropharyngeal Abscess in the Neonate}

Sir,

Retropharyngeal abscess (RPA) is very rare in the neonate. ${ }^{1,2}$ Herein, we report a neonate with RPA who had airway compromise and mediastinitis and was managed successfully.

A 28-day-old male baby was brought to the pediatric emergency services with complaint of fever, neck swelling and lethargy, with refusal to feed. Examination revealed a febrile, lethargic and sick looking neonate with palpable cervical lymph nodes of $1-1.5 \mathrm{~cm}$ size on the left side. USG of swelling on the left side of neck showed multiple enlarged hypoechoeic lymph nodes. CRP was positive and blood culture was sterile. Clinical diagnosis of lymph node abscess with sepsis was made and injection amoxyclav and amikacin were charted.

On day 3 of admission, baby developed severe respiratory distress. Air entry was markedly decreased on both sides, but there was no stridor. The baby was immediately intubated and showed dramatic improvement in respiratory distress and air entry. A repeat USG revealed retropharyngeal abscess. 